山्山 FRANÇAISE

$\gg \mathrm{DE}$

필 PÉDAGOGIE
Revue française de pédagogie

Recherches en éducation

182 | 2013

Connaissances et politiques d'éducation : quelles interactions?

\title{
Expériences de mixités vécues par les enseignants d'éducation physique et sportive : lien entre vigilance et équité sexuée
}

Experiences of coeducation lived by physical education teachers. Relation

between awareness and gendered equity

Catherine Patinet et Geneviève Cogérino

\section{(2) OpenEdition}

Journals

Édition électronique

URL : http://journals.openedition.org/rfp/4014

DOI : 10.4000/rfp.4014

ISSN : 2105-2913

Éditeur

ENS Éditions

Édition imprimée

Date de publication : 28 août 2013

Pagination : 93-106

ISSN : 0556-7807

Référence électronique

Catherine Patinet et Geneviève Cogérino, «Expériences de mixités vécues par les enseignants

d'éducation physique et sportive : lien entre vigilance et équité sexuée », Revue française de pédagogie [En ligne], 182 | 2013, mis en ligne le 28 août 2016, consulté le 10 décembre 2020. URL : http:// journals.openedition.org/rfp/4014; DOI : https://doi.org/10.4000/rfp.4014 


\section{Expériences de mixités vécues par les enseignants d'éducation physique et sportive : lien entre vigilance et équité sexuée}

\section{Catherine Patinet et Geneviève Cogérino}

La présente étude se propose de décrire, dans une perspective psychophénoménologique, les différentes expériences de mixités vécues par les enseignant-e-s lors des cours d'éducation physique et sportive (EPS). Derrière le vécu subjectif des enseignant-e-s opèrent des logiques intrinsèques qui participent à l'orientation d'une attention plus ou moins tournée vers l'équité. Les enseignant-e-s ne sont pas sans conscience pré-réfléchie des actes professionnels amenant à plus d'émancipation ou de renforcement des rôles sociaux conventionnellement attribués à un sexe. Mais cette conscience pré-réfléchie se traduit rarement en une véritable vigilance envers l'équité. La recherche montre également en quoi certaines formes de mixité sont des impasses pour être attentif à l'égalité des sexes.

Mots-clés (TESE) : éducation physique, éducation mixte, égalité des sexes, expérience professionnelle.

\section{INTRODUCTION}

La mise en place de la mixité en éducation physique et sportive (EPS) a été discutée au niveau professionnel dans les années quatre-vingt-dix à partir du constat des différences de réussite entre les filles et les garçons (Cleuziou, 1996, 2000). Dans le même temps, les recherches en EPS dans les pays anglosaxons (Evans, Davies \& Penney, 1996 ; Rǿnholt, 2002 ; Wright, 1997) et plus récemment en France (BonniotPaquien, Cogérino \& Champely, 2009 ; Cogérino, 2006 ; Couchot-Schiex \& Trottin, 2005 ; Guérandel \& Beyria, 2010 ; Verscheure \& Amade-Escot, 2007 ; Vigneron, 2006b) ont confirmé les travaux sociologiques et psychologiques français (Duru-Bellat, 1990, 1994, 1995 ; Mosconi, 1989) : I'institution scolaire met en jeu, sans que l'enseignant-e semble en avoir conscience, un mécanisme de socialisation sexuée. Les modalités langagières ou les contenus d'enseignement véhiculent de nombreux stéréotypes de sexe et contribuent à un curriculum caché. Les élèves, garçons ou filles, ne bénéficient pas d'opportunités d'apprentissage identiques. L'approche didactique des inégalités montre également dans le détail comment l'implication des élèves dans les tâches semble liée au type de savoir enseigné et au type de "direction d'étude " réalisée par l'enseignant-e (agencement des milieux didactiques et gestion au fil des séances de l'interaction des élèves à ces milieux). L'action didactique conjointe des enseignant-e-s et des élèves a des effets sur la construction de la différence des sexes en cours d'EPS (Verscheure, 2009). 
L'EPS est une discipline à connotation masculine et s'appuie sur des activités physiques sportives et artistiques (APSA), elles-mêmes différemment connotées par les enseignant-e-s et les élèves (Fontayne, Sarrazin \& Famose, 2001 ; Koivula, 2001). L'intégration des APSA comme support de la discipline a concouru à privilégier une "culture sportive " structurée autour d'un modèle de "puissance et de performance ", à valence masculine (Férnandez-Balboa, 1993 ; Théberge, 2000). Les enseignant-e-s choisissent un éventail restreint d'APSA, valorisent un modèle sportif compétitif peu attractif pour de nombreuses filles (Combaz \& Hoiban, 2008). Enfin, plusieurs enquêtes qualitatives (Guérandel \& Beyria, 2010 ; Vigneron, 2006a) montrent que les comportements des garçons sont l'étalon à partir duquel les enseignant-e-s gèrent les séances.

Ces travaux développent des modèles explicatifs où les discriminations ${ }^{1}$ opéreraient de manière discrète sans que les enseignant-e-s en aient conscience. Une approche psychophénoménologique de la mixité permettrait de décrire la subjectivité des expériences vécues par les enseignant-e-s et ainsi de caractériser leur attention lors des cours mixtes d'EPS. L'enjeu de la présente recherche est de décrire et de comprendre les liens chez les enseignant-e-s entre la mixité mise en place et les divers types de vigilance envers l'équité sexuée.

La revue de questions procède en trois temps. D'abord, la diversité des modes d'approche de la mixité en EPS sera rappelée. Puis l'équité sexuée sera définie au travers des différents modèles et catégories d'action établissant un enseignement équitable versus sexiste. Nous montrerons ensuite en quoi l'approche psychophénoménologique constitue une plus-value quant à l'investigation de la mixité dans l'enseignement scolaire et préciserons le concept d'attention.

\section{PRATIQUES ET REPRÉSENTATIONS DE LA MIXITÉ}

La mixité sexuée consiste à regrouper les élèves des deux sexes. Compte tenu des différences de ressources chez les élèves en EPS (différences entre garçons et filles dans les représentations de I'EPS et des APSA, dans l'expérience sportive, les qualités physiques, les attitudes), cette mixité ne faciliterait pas le travail des enseignant-e-s (Terret, Cogérino \& Rogowski, 2006). Les enseignant-e-s font face à ces difficultés ressenties par des stratégies qui renvoient à diverses conceptions des liens entre égalité et équité.
Très classiquement, on pourrait mettre en relation ces stratégies avec les problématiques de l'égalité de réussite, de l'égalité de traitement ou de l'équité dans le développement.

Les élèves du second degré sont dans un moment de fragilité par rapport à la construction de leur identité sexuée et ont développé, au travers de leurs propres expériences socioculturelles, des goûts et des compétences divers. La mixité constitue pour eux une expérience subjective particulière : celle du regard des autres, de même sexe ou non, et la prise de conscience d'appartenir à un groupe de sexe dominant ou dominé. La connotation masculine de l'EPS ainsi que les APSA masculines le plus couramment enseignées (sports collectifs, pratiques avec recherche de performance, etc.) ne favorisent pas l'intégration d'une majorité de filles qui intériorisent l'idée que leur place est d'être plutôt secondes.

Pourtant, Artus (1999) souligne que les enseignante-s confèrent un " caractère d'évidence » à la mixité. Elle serait perçue comme une forme de groupement "naturelle » qui génère des attitudes positives, le respect des différences, l'enrichissement mutuel, la répartition des rôles dans une tâche. Les enseignante-s expriment également les connotations sexuées attachées à certaines activités physiques (masculines pour les sports collectifs ou le combat ; féminines pour la gymnastique ou la danse ; neutres pour l'escalade, la natation ou l'athlétisme). Toutefois, l'apparition de la puberté chez les élèves provoque chez les enseignant-e-s des difficultés croissantes (Terret, Cogérino \& Rogowski, 2006) alors que le corps sexualisé est le plus souvent dénié lors des cours d'EPS mixtes (Liotard, 2000).

Quand on interroge les enseignant-e-s sur la mixité, ce qui semble les questionner en premier porte sur les formes de groupement des élèves à l'intérieur du cours : quand faut-il ou non démixer ? Cette préoccupation est d'autant plus forte que les enseignante-s d'EPS constatent au quotidien des différences de réussite et d'engagement en fonction du sexe. Se pose alors la question de la réussite des filles dans une discipline fortement masculine du fait des contenus d'enseignement d'origine « sportive ».

Différentes modalités sont proposées pour gérer la mixité des classes : la séparation des deux sexes, les groupes de niveau (les garçons sont le plus souvent dans les groupes forts), les groupes de besoin (réunis autour de niveaux d'habileté proches), les groupes affinitaires, les groupes hétérogènes avec ou sans aménagement (Davisse \& Volondat, 1987 ; Bordes, 2000). Ces 
regroupements sont plus pilotés par des « critères de viabilité " (Hauw, 2000) que par les enjeux de mixité ou d'équité. Ils permettent à l'enseignant-e de voir en un seul coup d'œil les événements du déroulement du cours et d'ajuster en permanence son intervention. Plusieurs auteurs déclarent que les particularités et la spécificité des APSA influent sur la constitution des groupes mixtes en EPS (Bordes, 2000 ; Terret, Cogérino \& Rogowski, 2006 ; Benhaïm-Grosse, 2007). Par exemple, dans les activités où interviennent fortement les caractéristiques morphologiques (sports de combat, rugby, escalade), le critère sécuritaire est prioritaire (en mettant ensemble des élèves de taille et de poids assez proches). Dans les activités requérant le partenariat (danse), les regroupements affinitaires (et donc généralement unisexués) prédominent.

En définitive, les enseignant-e-s, dans le feu de l'action du cours mixte, sont accaparé-e-s par d'autres soucis que celui de la mise en place ou du maintien de l'équité sexuée. Ils semblent se focaliser sur la constitution des groupes et ne pas interroger leurs propres choix didactiques, propos ou interactions. On peut alors se demander si les enseignante-s ont conscience des inégalités de traitement que rencontrent les élèves, celles-ci ayant été largement établies par les recherches procédant par observation dans les classes. II s'agit donc de s'interroger sur les conceptions des enseignant-e-s à l'égard de l'équité sexuée.

\section{MODÈLES D'ÉQUITÉ SEXUÉE}

Dans l'urgence et l'incertitude des microdécisions, les enseignant-e-s s'en remettent à des valeurs, des normes, des principes sur ce qu'ils-elles croient juste de faire pour assurer l'équité entre leurs élèves. Dans le champ de la sociologie de l'éducation, Crahay (2000), Dubet (2004), Dubet et Duru-Bellat (2004) clarifient plusieurs modèles d'équité, centrés sur l'égalité des chances, des résultats ou des opportunités d'accès. Davis (2003), Knoppers (1988) et Laberge (2004) prennent appui sur ces modèles et restituent le lien entre les conceptions d'un enseignement équitable et les représentations des enseignant-e-s d'EPS (relatives aux différences de ressources, de comportements et de résultats entre filles et garçons) :

- le premier modèle estime qu'il y a peu de différences, que les deux sexes sont équivalents. Cette "équité comme égalité de traitement "suggère que la même offre d'enseignement doit être donnée aux deux sexes. Par exemple, les élèves garçons et filles seront mélangés pour réaliser des équipes de sports collectifs mixtes hétérogènes sans prendre en compte les différences. Cette conception " méritocratique " situe la compétition scolaire comme juste et équitable : des savoirs identiques pour tous permettront aux meilleurs de se dégager car fournissant plus d'efforts. En réalité, cette égalité de traitement ne fait que renforcer les statuts de garçons dominants et de filles dominées car il s'agit là d'une compétition parfaite entre compétiteurs inégaux (Dubet \& Duru-Bellat, 2004, p. 105) ;

- le deuxième modèle, l'« équité comme égalité compensatoire ", présuppose que les filles sont déficientes (moins fortes, moins rapides, moins puissantes, moins impliquées) par rapport à la culture masculine. L'éducation compensatoire prend la forme d'avantages donnés aux filles (on propose ainsi des règles plus faciles ou des réussites qui amènent davantage de points que pour les garçons) ou de « handicaps » pour les garçons (partir de plus loin, manipuler un objet plus lourd). Ces pratiques compensatoires (comportements, choix de situations d'apprentissage ou propos de l'enseignant-e) constituent des " discriminations positives » ayant pour but d'atténuer les différences de ressources ou de résultats entre les deux groupes de sexe ;

- le troisième modèle, l'« équité comme égalité différentialiste ", reconnaît l'égale importance des différences et cherche à valoriser la diversité et l'hétérogénéité. L'enseignant-e crée alors deux groupes de sexe séparés, persuadé-e que la réussite des garçons est attribuable à leurs qualités motrices et à leur intelligence alors que les filles, peu motivées, sont responsables de leur échec. Ceci conduit à la ghettoïsation et au renforcement des stéréotypes. Ces trois premiers modèles d'équité contribuent à ce que les élèves intériorisent les rôles sociaux conventionnellement attribués à leur sexe ${ }^{2}$;

- enfin, le dernier modèle, l'« équité comme égalité dans la qualité du développement individuel » (Hadji, 2008), considère que garçons et filles sont semblables et différents, et doivent recevoir des opportunités de développement différentes mais de valeur équivalente. Ce modèle prend différentes formes: instauration du tutorat (le plus souvent par la constitution de dyades mixtes où les garçons conseillent les filles) ; intervention sur tout comportement ou attitude sexiste ; mise en place de contenus sollicitant chez les élèves l'adoption d'attitudes, de comportements, de capacités ou d'habiletés considérés comme typiques de l'autre 


\begin{tabular}{|l|l|}
\hline Catégories d'actions équitables & Catégories d'actions sexistes \\
\hline $\begin{array}{l}\text { L'absence de préjugés sexistes dans les comportements } \\
\text { ou verbalisations }\end{array}$ & L'affichage ou l'expression de préjugés sexistes \\
\hline $\begin{array}{l}\text { La mise en place de situations précisant la répartition } \\
\text { des rôles, limitant la domination d'un groupe de sexe }\end{array}$ & $\begin{array}{l}\text { La mise en place de situations ne précisant pas les } \\
\text { rôles places de chacun, permettant la domination } \\
\text { d'un groupe de sexe }\end{array}$ \\
\hline $\begin{array}{l}\text { La mise en place de situations permettant aux deux } \\
\text { sexes d'apprendre }\end{array}$ & $\begin{array}{l}\text { La mise en place de situations ne permettant pas à un } \\
\text { des deux sexes d'apprendre }\end{array}$ \\
\hline $\begin{array}{l}\text { L'ouverture des situations à des registres d'action } \\
\text { masculins et féminins }\end{array}$ & $\begin{array}{l}\text { L'exclusivité des situations à un registre d'action } \\
\text { typique d'un seul groupe de sexe }\end{array}$ \\
\hline $\begin{array}{l}\text { L'équilibre en quantité et en qualité des interventions } \\
\text { en faveur des deux sexes }\end{array}$ & $\begin{array}{l}\text { Le déséquilibre en quantité et en qualité de l'intervention } \\
\text { en faveur d'un seul sexe }\end{array}$ \\
\hline $\begin{array}{l}\text { La recherche d'un climat de classe rejetant toute forme } \\
\text { de stigmatisation* ou d'insultes sexistes }\end{array}$ & $\begin{array}{l}\text { La stigmatisation d'un-e élève ou d'un groupe d'élèves } \\
\text { par rapport à son sexe } \\
\text { La non-intervention face à une attitude ou une insulte } \\
\text { sexiste }\end{array}$ \\
\hline
\end{tabular}

Source : d'après Lee, Marks \& Byrd, 1994.

Note : * : la stigmatisation correspondant à la mise à l'écart d'un élève, ici en lien avec son sexe.

sexe. Par exemple, en danse, solliciter des modalités d'exécution qui alternent l'attaque du mouvement par une énergie explosive (registre plus masculin) avec un mouvement relâché (registre plus féminin) (Coltice, 2004). Ce modèle implique également des "mesures différenciatrices à visées correctrices" (Duru-Bellat, 1999, p. 23), puisque l'équité ne se conçoit qu'en fonction de la situation de chacun.

Au-delà des modèles d'équité qui traversent l'enseignement de I'EPS, comment définir des actions équitables ou sexistes ? Lee, Marks et Byrd (1994) affirment que les enseignant-e-s jouent un rôle dans la résistance au sexisme ou dans sa promotion. À partir de l'observation d'un enseignement mixte et unisexe concernant diverses disciplines scolaires, elles proposent de catégoriser le sexisme selon six formes typiques :

- le renforcement du genre défini comme la perpétuation des définitions sociales conventionnelles du genre avec le sexe d'appartenance ;

- l'incorporation des discriminations genrées au travers du langage, des textes littéraires, des archives historiques, des médias ;

- le renforcement des rôles de sexe où les filles sont systématiquement à leur désavantage ;
- la domination masculine, soit accordée par les autres, soit exercée par eux-mêmes ;

- la discrimination active ou dévalorisation des filles ;

- l'évocation explicite de la sexualité ou le traitement des garçons et des filles comme objet sexuel.

Ces catégories d'action contribuent à renforcer les définitions sociales conventionnelles du genre en lien avec le sexe d'appartenance. À l'inverse, l'équité sexuée est définie au travers de catégories visant à émanciper durablement des rôles sociaux conventionnellement attribués à un sexe. Nous avons transposé ce cadre pour les enseignant-e-s d'EPS (voir tableau 1).

\section{VIGILANCE ET ATTENTION}

Les travaux portant sur les pratiques et représentations de la mixité mettent en lumière le poids de la norme masculine, mais ils ne rendent pas compte de la subjectivité ${ }^{3}$ des acteurs, autrement dit de ce qui fait sens dans leur expérience concrète vécue. La psychophénoménologie se présente alors comme une démarche complémentaire des recherches jusqu'alors 
poursuivies ${ }^{4}$. La subjectivité des acteurs étant le fondement même de cette expérience, elle devient un objet valide de recherche. De plus, les recherches précitées nous laissent croire que les enseignante-s seraient sexistes (ou équitables) sans en avoir conscience. Dans le sens courant, avoir conscience de quelque chose, c'est avoir une représentation mentale claire de l'existence, de la réalité de telle ou telle chose. Pourtant, la langue anglaise distingue deux termes pour le mot " conscience ": « awareness" (que l'on pourrait traduire par le terme de vigilance) et « consciousness" (activité réflexive) (Depraz, 2002). Ceci nous permet de distinguer un premier niveau de conscience pré-réfléchie (immanente à l'action, incarnée, spontanée) d'une conscience plus réfléchie. La perspective psychophénoménologique (Vermersch, 1994) apporte à la fois un éclairage nouveau sur les pratiques professionnelles des enseignant-e-s en contexte mixte et une méthode (l'entretien d'explicitation) pour approcher leurs actions pré-réfléchies, ainsi que leur vigilance. En décrivant l'attention des enseignant-e-s lors des cours mixtes, et en la mettant en relation avec les catégories d'actions équitables ou sexistes, nous pouvons caractériser la vigilance à l'égard des inégalités de réussite des élèves.

L'attention est un objet dynamique qui apparaît par ses changements contrastés (Vermersch, 1998, 2002). Dans notre champ de conscience, un " thème attentionnel » est saisi de façon centrale, mais à tout moment des éléments périphériques peuvent passer au centre et le thème attentionnel initial passer à la périphérie. II est ainsi important de distinguer ce qui est visé par l'attention dans une vigilance particulière et par anticipation (« je m'attends à quelque chose ») de ce qui capte l'attention par des traits saillants au milieu d'autres informations (" je perçois quelque chose que je n'attendais pas particulièrement ") et qui peut ou non distraire de la visée initiale. Spontanément, un sujet décrit l'objet central de son attention (le " remarqué primaire »), ce dont il est le plus souvent réflexivement conscient. Mais il ne parle pas de ce qu'il a pris en compte de manière pré-réfléchie (les "remarqués secondaires ", les " co-remarqués", les " arrièreplans »). Décrire l'attention est donc tout à la fois décrire le «contenu » de l'attention (les « remarqués », ce à quoi on est attentif), les « actes » qui le médient (percevoir, raisonner, imaginer, se souvenir, décider) et en décrire le mouvement.

Ainsi, les questions centrales qui guident cet article sont les suivantes : quelle attention exercent les enseignant-e-s à l'égard des inégalités de réussite des élèves liées aux différences sexuées (attraits diffé- renciés à l'égard de l'EPS, diversité des ressources motrices, etc.) ? Considèrent-ils-elles ces différences comme allant de soi, si bien qu'ils-elles n'ont pas à chercher à lutter contre ? Ou bien dépassent-ils-elles leurs préjugés sexués pour exercer une vigilance à ce propos ? Comment ces diverses « vigilances " à l'égard des discriminations sexuées varient-elles selon les différentes expériences vécues de mixité ?

\section{MÉTHODOLOGIE}

L'échantillon a été constitué de vingt-quatre enseignant-e-s de collège de façon à équilibrer : le nombre d'hommes et de femmes ; le nombre d'années d'ancienneté dans l'enseignement (moins de 5 ans, de 5 à 10 ans, 10 à 20 ans, plus de 20 ans) ; le type d'établissement (« normal », REP, zone sensible, rural) ; le niveau de diplôme (CAPEPS interne, externe, agrégation interne, externe) - voir annexe 1. Le choix de ces critères pour notre panel reprend les variables de Terret, Cogérino et Rogowski (2006) qui montrent une différenciation des représentations de la mixité selon les sexes, les générations et les diplômes.

Le recueil de données repose sur la technique d'« entretien d'explicitation » (Vermersch, 1994). La psychophénoménologie s'intéresse à la subjectivité et cherche à s'en informer en demandant au sujet de décrire le contenu de son expérience. L'entretien d'explicitation (EdE) permet le récit d'une expérience passée et sa description, pas à pas, par un-e enseignant-e invité-e à rendre à nouveau présent à sa conscience (par un discours en première personne) cette expérience passée.

L'EdE comporte un certain nombre d'exigences. Tout d'abord, il est indispensable que les données se rapportent à un vécu de référence (ce qu'il s'est passé " ce jour-là, à ce moment-là ") pour éviter tout discours sur des généralités. La deuxième exigence est que l'interviewé-e rende à nouveau présent à sa conscience son vécu. Tout au long de l'EdE, le chercheur doit faire en sorte que persiste cette qualité d'évocation qui est la condition d'un accès à un vécu passé. Une des clés est de faire appel à une mémoire concrète et de guider l'enquêté-e vers une évocation sensorielle : ce qui est vu, entendu, perçu. Une installation dans le contexte du moment choisi est nécessaire pour que la personne interviewée ne donne pas des explications mais explicite, c'est-à-dire se tourne vers elle-même, vers son monde intérieur, et remobilise un vécu passé. Enfin, l'EdE repose sur un 
Tableau 2. Catégories descriptives des entretiens d'explicitation (EdE)

\begin{tabular}{|l|l|}
\hline Catégorie & \multicolumn{1}{c|}{ Indicateurs } \\
\hline $\begin{array}{l}\text { Thème } \\
\text { attentionnel }\end{array}$ & $\begin{array}{l}\text { Donné comme intention : « mon souci est... », « je cherche à... », « je veux... », « ce qui } \\
\text { est important c'est » } \\
\text { Inféré à partir des actes et des contenus des actes }\end{array}$ \\
\hline $\begin{array}{l}\text { Actes qui } \\
\text { précisent } \\
\text { le thème } \\
\text { attentionnel }\end{array}$ & $\begin{array}{l}\text { Actes perceptifs : « je vois », « je regarde », «j'entends », « je sens » } \\
\text { Actes cognitifs : « je me dis »; les actes décisionnels : « je décide », « je choisis », « je } \\
\text { prère " }\end{array}$ \\
\hline $\begin{array}{l}\text { Contenus métacognitifs } \\
\text { attentionnels }\end{array}$ & $\begin{array}{l}\text { Remarqués primaires, remarqués secondaires, co-remarqués } \\
\text { Prégnances internes ou externes qui vont modifier ou non le thème principal }\end{array}$ \\
\hline Croyances & $\begin{array}{l}\text { Opinions, valeurs, dogmes, représentations } \\
\text { Préjugés sexistes } \\
\text { Croyances liées aux gestes professionnels }\end{array}$ \\
\hline Émotions & " je suis inquiet », « je suis content », «ça m'énerve » \\
\hline Arrière-plans & $\begin{array}{l}\text { Verbalisations concernant les savoirs d'action ou les données des expériences passées. } \\
\text { Les arrière-plans peuvent se situer à des horizons temporels très différents. }\end{array}$ \\
\hline
\end{tabular}

questionnement non inductif qui dirige l'attention de l'interviewé-e vers des aspects de l'expérience dont il-elle n'a pas la conscience réfléchie ni les catégories pour y réfléchir. Ainsi le chercheur veille à reprendre les mots, les verbes, le vocabulaire de l'enseignant-e. La technique de questionnement qui suit le moment de focalisation se caractérise par une non-directivité du point de vue du contenu évoqué et en même temps par un guidage précis cherchant à élucider le déroulement de l'action, en encourageant la description et en bannissant la question du " pourquoi ? ». Les relances se situent à la fois sur un axe chronologique pour décrire le début, le moment et la fin (avec des questions comme : "Comment tu as commencé ? et ensuite ? ») et sur différentes échelles de granularité de la description (par exemple : « Et quand tu fais... qu'est ce que tu fais ? "). Les relances cherchent à identifier les prises d'informations, les décisions, les effectuations (par exemple : «Et quand... à quoi tu sais que ? et là, à quoi es-tu attentif ? ") et à mettre au jour les différentes structures feuilletées de l'attention (par exemple : “ Et tout en continuant à..., qu'est ce que tu fais aussi ? »).

L'acte d'évocation a été amorcé à partir de la suggestion suivante: " Je te propose, si tu es d'accord, de retrouver un moment de cours dans lequel tu t'es senti-e capable de faire apprendre ensemble les filles et les garçons ". C'est donc l'enseignant-e, et lui-elle seul-e, qui a choisi la situation évoquée. Suite à cette proposition, l'enseignant-e prend le temps de focaliser sur un moment remarquable, de son point de vue, et l'EdE se déroule comme décrit précédemment à partir de ce premier récit. Après cette première évocation positive, une deuxième suggestion est de «[...] retrouver un moment de cours dans lequel tu ne t'es pas senti-e capable de faire apprendre ensemble les filles et les garçons ». Un deuxième moment d'explicitation est alors exploré à partir d'une évocation négative.

L'étude a ainsi rassemblé quarante-huit récits explicités dont vingt-quatre vécus positivement et vingtquatre vécus négativement. Seuls trente-neuf moments explicités ont été conservés. Nous avons éliminé tout entretien ou partie d'entretien sortant de l'évocation du moment décrit et portant sur des souvenirs, des généralités, des classes de vécu. L'identification des thèmes attentionnels lors des moments évoqués nous permet de répondre aux questions de notre recherche.

À l'issue des deux temps d'explicitation portant sur deux récits, un troisième temps d'entretien semi-directif a permis d'approcher les représentations personnelles des inégalités homme-femme, les modèles de justice sexuée (en général et à l'école) et le vécu personnel, les 
expériences de mixité ${ }^{5}$. L'engagement en fonction des valeurs, des idéaux portés par le système de représentations de l'enseignant-e peut en effet constituer un arrière-plan explicatif des décisions et actions prises.

Pour le traitement des données issues des moments d'EdE, nous avons adapté à notre propre objet d'étude les catégories descriptives provenant des recherches effectuées en psychophénoménologie (Mouchet, 2003). Six catégories descriptives ont été retenues (voir tableau 2) :

- les thèmes attentionnels ;

- les actes qui précisent le thème attentionnel ;

- les contenus attentionnels (qui permettent de décrire ce qui va alerter ou éloigner de la vigilance envers l'équité) ;

- les croyances et préjugés sexistes ;

- les émotions révélatrices d'enjeux, de valeurs, de sens identitaire ;

- les arrière-plans à la situation.

Grâce à ces catégories, la description de chaque moment vécu permet de répondre à la question : à quoi les enseignant-e-s d'EPS font-ils-elles attention quand ils-elles font cours en mixité 6 ? Puis une interprétation a été possible de leur niveau de vigilance envers l'équité sexuée : leur attention est-elle dirigée ou non vers des actions équitables (voir tableau 1)?

\section{RÉSULTATS}

Si l'expérience de mixité est toujours singulière pour chaque moment, notre étude permet de montrer des régularités dans les expériences mixtes vécues qui n'amènent pas à la même vigilance. Le traitement des trente-neuf moments d'explicitation a permis de constituer quatre catégories d'expériences subjectives de la mixité. Elles mettent l'accent sur les modalités d'attention poursuivies et les critères d'identification réveillant ou éloignant la vigilance des enseignant-e-s. Chacune d'entre elle montre des différences dans les dynamiques attentionnelles, ainsi que dans les formes d'équité poursuivies. Ces catégories d'expériences ont été dénommées: les mixités ensemble-séparées, les mixités banalisées, les mixités recherchées et les mixités réfléchies. Elles rassemblent un nombre variable de moments explicités : sept pour la première, treize pour la seconde, seize pour la troisième et sept pour la dernière. Quatre moments décrivent le passage d'une catégorie de mixité à une autre et sont donc indexés dans deux catégories. Étant donné la taille de l'échantillon, cette répartition n'est donnée qu'à titre indicatif. Rappelons que ce n'est pas l'enseignant-e qui fait l'objet de la catégorisation mais bien l'expérience évoquée. La comparaison (que nous ne développerons pas dans cet article) pour un-e même enseignant-e des moments vécus positivement et négativement montre que ces expériences subjectives de la mixité diffèrent d'un contexte d'enseignement à l'autre.

\section{La double attention des mixités ensemble- séparées}

Le terme de mixité "ensemble-séparée » est emprunté à Goffman (1977). Soit il s'agit d'une séparation des garçons et des filles initiée par l'enseignante, soit il s'agit de la séparation tacite des élèves en deux espaces ségrégués que l'enseignant-e laisse faire : la mixité est alors une "mixité en apparence". En général, la séparation des deux sexes est justifiée par les enseignant-e-s par le fait qu'il y a trop de différences entre garçons et filles pour une pratique ensemble. Tout en restant réservées sur la taille de l'échantillon, nous constatons que les récits de ces mixités «ensemble-séparées » portent majoritairement sur des sports collectifs, aux connotations masculines. Certain-e-s enseignant-e-s concerné-e-s expriment alors des intentions d'éducation compensatoire, pour faire rattraper le niveau aux filles.

Les premiers critères donnés dans les évocations mentionnent le groupe des garçons qui fonctionne seul et le groupe des filles en échec : « Bon, là, les garçons, hop, c'est parti, je ne reviens pas [...] et les filles, ça n'a pas du tout marché. " (Michel, football). Peu de prénoms d'élève sont mentionnés. Les contenus attentionnels sont établis par comparaison entre les deux groupes de sexe : "Oui, en périphérique, dans mon dos, je sens que les filles ne contrôlent pas le ballon et que les garçons se font des grandes passes. " (Michel, football).

L'attention est déséquilibrée vers les garçons ou vers les filles. L'enseignant-e est le plus souvent partagée entre ces deux groupes, agissant «ici » avec un groupe de sexe, mais restant toujours «là-bas " en actes attentionnels de surveillance ou de guidage avec l'autre groupe: « Je lance ici, je vais là-bas, je fais des observations ici, et je reviens ici. Quand je siffle, ça tourne, même si je suis là-bas. " (Michel, football). Les signes d'intervention sur l'autre groupe sont différenciés selon qu'il s'agit des garçons ou des filles. Pour les garçons, c'est le risque de désordre qui prime: 
des cris, un garçon qui sort du terrain, un changement d'ambiance par rapport au jeu demandé. Pour les filles, ce sont les signes d'inactivité. Les verbalisations sont accompagnées de nombreux préjugés : «Alors, tout de suite, ils [les garçons] percutent. Les filles, c'est plus difficile [...] ça ne leur correspond pas. " (Michel, football). On peut qualifier ces préjugés de sexistes dans la mesure où ils étendent l'appréciation formulée à tout un groupe de sexe, et où il ne s'agit pas d'un constat précis (effectué à propos de personnes identifiées) mais d'une formule générale. La norme masculine est valorisée, par des attentes de mouvement, de vitesse, de combativité. L'émotion provoquée par la vision de l'échec des filles est également perceptible, ce qui amène à des micro-stratégies de renoncement d'intervention à leur égard : «Après, je me suis dit : c'est pas la peine que j'intervienne... auprès des filles pour dire "fais pas ci, fais pas ça" ou "fait ça", parce que je me dis : bien, si cela n'apparaît pas là, c'est que ce n'a pas été intégré et que ce ne le sera pas. " (Basile, tchoukball7). À l'inverse, les difficultés de quelques garçons sont mieux tolérées et déclenchent des stratégies de régulation pour les remettre en confiance: "Oui, j'interviens et je lui [un garçon] dis: "pourquoi tu tires pas là ?" " (Basile, tchoukball).

Dans la mixité ensemble-séparée, les enseignant-e$s$ ne peuvent mettre ensemble les garçons et les filles étant donné leurs différences, et les deux groupes ségrégués prouvent au quotidien la fiabilité de cette thèse. La vision en deux groupes de sexe favorise une lecture de l'activité de l'élève biaisée par des attentes sexuées et génère une double attention rendant difficile la recherche d'indices explicatifs des comportements des élèves.

\section{La faible vigilance envers l'équité sexuée des mixités banalisées}

La mixité est banalisée quand les élèves cohabitent sans aménagement particulier. Elle n'est pas un choix délibéré de la part de l'enseignant-e qui a parfois d'autres préoccupations, comme lancer le cours ou assurer la sécurité. La conduite du cours est d'ailleurs la centration attentionnelle principale de cette catégorie d'expériences vécues (3 récits sur 4 de cette catégorie d'expériences). La mixité banalisée repose sur la croyance en une mixité naturelle liée à l'équivalence ou à la complémentarité des sexes. Adèle (volley-ball) décrit ainsi un tutorat mixte spontané : "C'est vrai qu'ils se sont amenés à d'eux-mêmes se mélanger. »
Cette logique intrinsèque de " conduire le cours pour des élèves » donne une illusion d'égalité de traitement. Mais les contenus d'enseignement décrits appartiennent en grande majorité à un registre masculin, y compris dans une activité comme la danse: «On part en courant et puis on pile l'un devant l'autre [...] je le prends par l'épaule, et vlan! Je le mets par terre [...]. J'attrape et j'envoie valdinguer. » (Audrey, danse). L'intention est ici d'intéresser les garçons en restant conforme à leurs stéréotypes. L'enseignante commente la situation en évoquant une façon de moduler l'énergie gestuelle sur un registre très " tonique " (courir vite et s'arrêter brusquement, jeter l'autre au sol, l'envoyer "valdinguer ») ; autant d'éléments qui s'éloignent quelque peu de l'image féminine associée à la gestualité dansée : gestes doux, aériens, un peu mièvres... Les mixités banalisées font alors craindre une centration exclusive de l'attention vers les garçons: " Je pense à lui à différents moments, déjà, je le vois faire, je le vois accepter de faire l'improvisation, de jouer le jeu [...]. Et puis je vois Émile... Et je le regarde lui, pour voir ce qu'il fait, et je le trouve super. Et je lui dis. " (Audrey, danse).

Quand les descriptions différencient les filles et les garçons, elles empruntent des critères proches des stéréotypes. Les filles sont lentes, n'osent pas s'imposer, ne font aucun effort, quand les garçons sont forts, s'amusent, manquent de maîtrise. Néanmoins, si la vigilance envers l'équité sexuée est faible, des signes d'alerte existent qui peuvent agir comme déclencheurs de recherche d'équité. Ces signes sont toujours issus d'une lecture plus approfondie des premiers signes sexués. Lors des EdE, les enseignant-e-s ont décrit fréquemment l'activité des filles par l'expression «le ballon, elles s'en débarrassent ». En utilisant ce terme "se débarrasser ", les enseignant-e-s précisent qu'il s'agit plus de se défaire de la balle que de faire une passe. Pourtant, en y regardant de plus près, l'identification du geste "se débarrasser » n'a pas tout à fait la même connotation dans tous les cas.

Pour Loïc, le geste est accompagné d'un préjugé négatif envers les filles : "Elle s'en débarrasse, mais pas parce qu'elle ne sait pas quoi en faire mais parce qu'elle ne veut rien en faire. " (Loïc, rugby). Si les filles se débarrassent de la balle, c'est par manque de motivation. Loïc se met alors en colère et les exclut du jeu, en disant qu'elles empêchent les garçons de jouer. L'attention s'oriente de façon nette vers leur stigmatisation.

Pour Audrey (ultimate 8 ), le geste " se débarrasser 》 est interprété différemment. Les filles ont peur des défenseurs garçons : «Elles s'en débarrassent, elles 
ne cherchent même pas dans la peur du défenseur... Ben, le défenseur arrive, je lance le frisbee, je crie. Voilà [rire]. » Audrey prend alors conscience qu'il faut mettre les filles en confiance et précise à nouveau les règles permettant d'éviter un contact trop violent : "Le défenseur, tu sais que tu ne peux pas t'approcher autant ou arracher le frisbee des mains, donc tu as le temps. " Puis elle fait passer deux fois de suite les filles et oriente toute son attention sur le respect des règles.

En résumé, sans être sans conscience des différences entre filles et garçons d'un point de vue préréfléchi, les enseignant-e-s sont, dans ces moments de mixité banalisée, en absence de véritable vigilance d'équité sexuée et restent perméables aux stéréotypes de sexe. Peuvent exister néanmoins des éveils attentionnels vers une instauration de l'équité. Ceux-ci ont toujours comme point de départ une interprétation des comportements des filles en lien avec la domination des garçons.

\section{Une vigilance plus tournée vers la recherche " à tout prix » de la mixité}

Dans cette configuration, l'enjeu pour l'enseignant-e est que les filles et les garçons pratiquent ensemble. Différentes micro-stratégies peuvent coexister : la négociation accompagnée d'un discours sur la complémentarité des sexes, la proposition de règles aménagées, la suggestion faite aux garçons d'apprendre aux filles ce qu'ils savent faire. Dans les critères d'identification, le moindre signe d'interaction garçon/fille est interprété comme un signe de mixité réussie : "Donc là, je fais de plus en plus attention à qui va se mettre avec qui et donc là je me souviens que j'en ai deux qui, garçons et filles qui se sont mis ensemble [...] ben, je me dis "bon ! [...] en voilà deux". " (Basile, lutte).

Les formes d'équité présentes correspondent souvent, lors des jeux d'équipe avec ballon, à des aménagements de règles qui avantagent les filles: " J'ai imposé que le but des filles compte triple, pour vraiment essayer de survaloriser le but des filles." (Éric, handball). L'enseignant-e a conscience que les garçons résistent le plus souvent à l'instauration de ces règles. L'attention se focalise alors sur les remarques négatives qu'ils font aux filles quand elles ne réussissent pas. La recherche de mixité est accompagnée d'un intérêt attentionnel visant à contrôler les garçons, à les modérer : « Je le [Maximilien] vois plutôt à faire des reproches quand les filles ne rattrapent pas une passe ou quoi que ce soit [...]. Je lui dis : "il faut que tu t'adaptes à elles, que tu leur fasses une plus petite passe". » (Audrey, handball). Les enseignant-e$\mathrm{s}$ cherchent également à voir si les règles favorisent l'activité des filles : «Ce que je regarde... Alors, j'ai des indicateurs... c'est-à-dire le nombre de fois où les filles ont touché la balle, si elles déclenchent des tirs. » (Éric, handball).

Parfois, s'instaure entre certains garçons et l'enseignant un jeu subtil où les garçons font semblant de répondre aux attentes de ce dernier : "Par moment, les garçons, ils arrivent à se dédouaner en disant : "on a fait des passes". Et la fille, elle sert de relais " (Éric, handball). Les enseignant-e-s perçoivent le manque de confiance des filles qu'ils-elles interprètent comme un renoncement volontaire. Néanmoins, leur jeu est accompagné d'encouragements sur leurs chances de réussite : "C'est de leur faire prendre confiance, de les encourager à prendre leurs chances, à tirer, parce qu'on les voit, des fois, elles sont toutes seules devant le but mais elles préfèrent passer leur balle à un garçon. » (Éric, handball).

Beaucoup d'enseignant-e-s prennent conscience des limites de ces aménagements compensatoires: sentiment d'injustice pour les garçons, échec et sentiment d'incompétence pour les filles et renforcement des stéréotypes de sexe. L'enseignant-e est alors dans une situation inconfortable, qu'il-elle masque soit en restant ferme sur la règle donnée, soit en procédant à de petits arrangements avec certains garçons, les autorisant, par exemple, à aller marquer à la dernière minute du jeu. Dans certaines descriptions, l'enseignant-e impose la mixité. Celle-ci est d'autant plus brusquée qu'elle renvoie à des activités avec contact corporel comme la danse ou la lutte. L'enseignant-e perçoit alors des signes de refus de pratiquer en groupe mixte (mouvements de recul, verbalisations de désapprobation, demandes explicites de pratiquer en groupe non mixte). Mais il-elle persiste le plus souvent à faire travailler en mixité et refuse de prendre en compte le rapport au corps sexué.

En résumé, dans les mixités recherchées, l'attention est essentiellement centrée sur la nature des interactions entre garçons et filles, au travers de règles instaurées. Les enseignant-e-s oscillent entre mettre en confiance les filles et convaincre les garçons que le jeu avec les filles est souhaitable et possible. La vigilance est tournée vers l'instauration du jeu en mixité plus que vers la recherche et la prévention de risques discriminatoires liés au jeu en mixité. Pourtant, les limites de ce type de mixité sont intuitivement perçues. 


\section{La fragilité des éveils attentionnels vers l'équité dans les mixités réfléchies}

Dans cette configuration, l'attention de l'enseignante s'oriente vers la recherche d'indicateurs liés aux apprentissages des filles et des garçons. Le recours à l'égalité compensatoire est plus réfléchi : plutôt que de donner davantage de points à une action réalisée par une fille (comme l'exemple précédent d'Éric où le but compte triple en handball pour les filles), les enseignant-e-s proposent de donner des pouvoirs différenciés de jeu aux garçons et aux filles (par exemple en protégeant les filles porteuses du ballon ou en limitant le dribble des plus forts). Les descriptions des contenus attentionnels parviennent à un grain de description plus fin. Une double veille attentionnelle est perceptible dans cette catégorie d'expérience : surveillance de l'engagement physique des garçons et sollicitation des filles pour qu'elles osent prendre leur place dans l'activité. Les conditions de la mixité sont ainsi davantage questionnées. Avant tout, l'enseignant-e décode de manière approfondie l'activité des filles et des garçons et le risque de sexisme de la situation. Il-elle décide ensuite d'intervenir pour accompagner les filles, montrer aux garçons qu'il-elle désapprouve leur comportement sexiste, rappeler la règle, la faire appliquer ou la changer.

Bertrand a pour thème attentionnel principal la supervision du jeu d'une équipe de trois élèves de $6 \mathrm{e}$, une fille et deux garçons, au cours d'un match de basket-ball. II remarque que les garçons jouent ensemble, font beaucoup d'échanges notamment sous la cible et qu'ils ne passent "presque pas " la balle à Jeanne. Bertrand identifie un problème de "capture " du ballon par Jeanne mais affirme que les garçons ont la possibilité de «moduler » la passe. Cet arrière-plan nous paraît important à mettre au jour car il prouve que, pour Bertrand, les garçons doivent exercer leur responsabilité dans le jeu avec une fille plus faible. Un nouvel élément intervient qui réoriente l'attention : la faible réussite au tir des garçons. Ceci déclenche chez Bertrand une intervention en direction des garçons : " Je leur ai dit : "vous jouez tout seuls les garçons". " L'attention s'infléchit alors vers le souci de voir un jeu plus collectif et Bertrand vérifie que les garçons passent davantage la balle à Jeanne. C'est alors que Jeanne tire et marque. Ce tir réussi est déclencheur d'un double intérêt : l'accompagnement de sa réussite pour la mettre en confiance et la démonstration aux garçons que leur attitude sexiste n'était pas efficace : "Je m'entends dire : "vous voyez les garçons, ça marche." [...] et Jeanne..., le terme exact, je ne sais plus. Peut-être : "tu vois, il faut y aller", ou "vas-y". "
Lors du temps d'échange après le match, la verbalisation concerne l'efficacité de Jeanne comme la preuve pour elle qu'elle est capable d'y arriver et pour les garçons qu'ils doivent lui faire confiance : "Les garçons, vous voyez, moi je jouerais dans l'équipe avec Jeanne, je lui donnerais la balle beaucoup plus souvent. "L'enseignant, en même temps qu'il affirme publiquement les potentialités des filles, dénonce subtilement l'attitude sexiste des garçons.

S'il n'y a pas une réelle vigilance à émanciper durablement les élèves de leurs stéréotypes sexués, on peut estimer que, lorsque les enseignant-e-s sont vigilants à la fois à contrôler les garçons et à aider les filles, ils-elles leur font faire un " pas en avant " vers l'émancipation des rôles sociaux conventionnellement attribués à un sexe. Toutes les descriptions de mixités réfléchies montrent des conditions favorables à sa mise en place. Il s'agit le plus souvent de moments de cours où l'enseignant-e peut focaliser et maintenir en prise son attention sur quelques élèves. La vigilance envers l'équité sexuée paraît ainsi difficile au regard des nombreuses préoccupations des enseignant-e-s.

\section{DISCUSSION}

Les limites de l'étude tiennent à l'effectif relativement restreint d'enseignant-e-s ayant formulé ces récits de pratique, questionné-e-s dans le cadre de la technique de l'EdE. Cependant, il y a là un nombre consistant d'entretiens dans le cadre d'une approche exploratoire et qualitative. Sur les trente-neuf moments explorés en EdE, dix-sept activités physiques sont représentées. Dix-neuf récits relèvent de neuf activités physiques traditionnellement connotées du côté du masculin, douze de six activités traditionnellement attribuées aux deux sexes, huit de deux activités traditionnellement connotées du côté féminin (voir annexe 2). Ce déséquilibre dans les choix d'activités évoquées par les enseignant-e-s pourrait laisser penser que ces dernier-e-s activent prioritairement, lors de la mise en évocation, des " expériences " relevant d'APSA fortement contrastées en terme de connotations et de stéréotypes sexués. Cette hypothèse est à relativiser au regard de la préférence attribuée aux sports collectifs par les enseignant-e-s : $39 \%$ les désignent comme " activité incontournable " lors de l'enquête de la Direction de l'évaluation, de la prospective et de la performance (DEPP) de 2005-2006 (BenhaïmGrosse, 2007). Lors de cette même enquête, il a été demandé aux enseignant-e-s, pour chaque famille 
d'activités, d'une part, s'ils-elles considèrent la mixité comme enrichissante pour les élèves, et d'autre part si la mixité leur pose des difficultés. Pour les sports collectifs de grands terrains et les sports de combat, la mixité est difficile à mettre en œuvre et rarement enrichissante. Pour ces activités, les enseignant-e-s préfèrent donc les groupes non mixtes. Ceci pourrait expliquer le nombre important d'activités masculines dans le groupe "mixité séparée ». Certaines activités ont le statut d'activité enrichissante mais difficile à mettre en œuvre (il faut être courageux pour la mettre en œuvre, mais les efforts de l'enseignant-e sont payés par des situations enrichissantes pour les élèves). C'est le cas des sports collectifs de petit terrain et des activités physiques d'expression, fortement représentés dans notre étude. La question initiale pour faire entrer en évocation les enseignant-e-s (" un moment de cours dans lequel tu t'es senti-e capable de faire apprendre ensemble les filles et les garçons ") renvoie à un sentiment de compétence qui a pu orienter le choix de l'évocation vers des activités difficiles et enrichissantes.

De fait, lors des moments explicités, il est probable que les garçons aient pu davantage montrer leurs compétences ou leur domination, avoir la possibilité d'aider les filles, être dans un rôle de tuteur dans ces activités en territoire masculin. La question qui peut alors se poser concerne la diversité des activités décrites. N'aurait-on pas obtenu d'autres résultats avec des descriptions portant sur des activités plus diverses ? Les centrations attentionnelles, les micro-stratégies ne seraient-elles pas sensiblement différentes? Ceci offre de nouvelles perspectives de recherche pour approfondir le lien entre la vigilance envers l'équité sexuée et le territoire masculin ou féminin des activités.

II resterait à clarifier le rôle que les contextes professionnels d'exercice (établissements en contexte classique, en zone d'éducation prioritaire [ZEP] ou en réseaux ambitions réussite [RAR] ...) et les histoires de vie des enseignant-e-s peuvent avoir sur leur vigilance envers l'équité sexuée en EPS. Ceci nécessiterait de s'engager dans une recherche qualitative à grande échelle.

D'autre part, si l'EdE parvient à reconstruire une partie des expériences vécues, il ne peut permettre d'accéder à la réalité concrète des pratiques effectives : l'étude proposée se présente bien comme une démarche complémentaire des recherches comportementales, en adoptant le point de vue du sujet en première personne, ce qui permet d'éclairer différemment la mixité en EPS. Les microdécisions effectuées dans l'urgence apparaissent erratiques à l'observateur extérieur, alors que l'EdE donne l'opportunité de saisir la " logique intrinsèque propre à chaque moment vécu ». Celle-ci, bien que difficilement conscientisable par le sujet, est soumise aux aléas de la situation, à ses propriétés dynamiques, à son imprévisibilité.

Le contexte de mixité créé par l'enseignant-e lui renvoie des indices qui vont soit renforcer ses préjugés sexistes, soit lui permettre de lire finement l'interaction entre les filles et les garçons. Cette lecture de l'activité adaptative des élèves (perceptible quand l'enseignante parvient dans l'EdE à des indices de granularité fine), de leur interaction, du risque de discrimination, peut réveiller une vigilance relative à l'équité. Alors que l'absence de cette lecture enferme dans les préjugés et les stéréotypes. Ainsi, quatre catégories d'expériences différentes dans les attentions et les modèles d'égalité sous-jacents ont été dégagées :

- les mixités ensemble-séparées reposent sur les attentes de deux mondes hiérarchisés, féminin et masculin. L'attention des enseignant-e-s est tiraillée entre filles et garçons, ce qui ne favorise pas leur capacité d'analyse fine des comportements des unes ou des autres;

- dans les moments de mixités banalisées, les enseignant-e-s regardent les inégalités sans les voir, préoccupé-e-s par le cours à conduire. Ils-elles ont l'illusion de pratiquer un même enseignement, d'offrir une " égalité de traitement » aux deux sexes, alors qu'ils-elles sont plus attentifs à des contenus masculins ;

- les mixités recherchées ont pour visée attentionnelle principale de " faire jouer ensemble " garçons et filles. Si les règles de discrimination positive sont expérimentées, dans cette "équité comme modèle compensatoire ", les critères retenus concernent le plus souvent la bonne ambiance de classe plus que la réussite dans les apprentissages ;

- lors des mixités réfléchies, l'enseignant-e déplace son attention entre aider les filles dans leur engagement et contrôler les garçons dans leurs possibles attitudes sexistes. Ils-elles questionnent davantage les conditions de la mixité en lien avec une éthique éducative où l'équité est conçue comme "égalité dans la qualité du développement".

Lors des EdE, les enseignant-e-s ont souvent pris conscience de contenus attentionnels montrant un risque discriminatoire lors de l'expérience vécue. Cette prise de conscience nous semble une piste à privilégier dans la formation des enseignant-e-s en 
développant des techniques d'analyse de pratiques. Si certains enseignant-e-s ont réalisé ces prises de conscience lors des EDE effectués, celles-ci ont été immédiatement gommées par des justifications essentialistes portant sur les différences sexuées entre les élèves. Peut-on alors penser la formation des enseignant-e-s sans éclairer la question des différences, et sans débat éthique sur les questions d'égalité ?

D'autre part, si des éveils attentionnels vers l'équité existent bien, on ne peut parler de véritable vigilance à son propos. Être vigilant, c'est à la fois être sur ses gardes, prêt à être réveillé par un danger imminent, mais aussi avoir la capacité de maintenir son atten- tion durablement sur un même objet. C'est bien sur cette question du mouvement de l'attention qu'il nous semblerait utile d'approfondir la recherche : quels sont les facteurs explicatifs de l'éveil, du maintien en prise ou de la distraction par rapport à une centration attentionnelle amenant à plus d'équité sexuée en EPS ?

Catherine Patinet cathythierry.patinet@wanadoo.fr Université Claude-Bernard, CRIS

Geneviève Cogérino genevieve.cogerino@univ-lyon1.fr Université Claude-Bernard, CRIS

\section{NOTES}

1 La discrimination prend ici le sens d'un traitement inégal et défavorable aux filles.

2 On trouvera dans l'ouvrage de Guérandel et Beyria (2010) de nombreux exemples issus d'une approche ethnographique, et fortement convergents avec ceux évoqués ici.

3 La subjectivité est ce que le sujet peut comprendre et dire de son expérience.

4 Une autre approche de la subjectivité est privilégiée par le « cadre théorique du cours d'action "(Theureau, 2004). L'intérêt de la méthode réside dans le fait d'articuler les traces de l'activité (par la vidéo) et les verbalisations a posteriori. Mais le risque est que le sujet face à ses images se positionne sur un discours " en seconde personne ", ce qui favorise dans les verbalisations ainsi provoquées l'emploi de généralités ou de justifications. L'entretien d'explicitation nous permet également d'explorer un nombre d'expériences plus important.
5 Exemples de questions guides : «De façon générale, que penses-tu du débat sur l'égalité entre les hommes et les femmes? "; "La mixité te semble-t-elle un enjeu essentiel de l'EPS ? "; "Quand tu étais élève, comment as-tu vécu la mixité au travers des cours d'EPS ? ".

6 Une catégorisation en huit thèmes attentionnels et modes d'intervention poursuivis en contexte mixte a été possible: " conduire le cours ", " contrôler les garçons ", « intéresser les garçons ", " aider les filles ", " exclure les filles ", " faire jouer ensemble les filles et les garçons ", " instaurer l'équité ", " accompagner la gêne occasionnée (ou le risque de gêne) par le corps sexualisé ».

7 Le tchoukball est un sport collectif qui se joue sur une cible qui renvoie le ballon dans la surface du jeu.

8 L'ultimate est un sport collectif qui se joue avec un frisbee.

\section{BIBLIOGRAPHIE}

ARTUS D. (1999). La mixité : illusion égalitaire en éducation physique et sportive ? Son étude à travers les représentations des enseignants et des lycéens. Thèse de doctorat, sciences et techniques des activités physiques et sportives, université de Poitiers.

BENHAÏM-GROSSE J. (2007). Image du sport scolaire et pratiques d'enseignement au lycée et au collège, 20052006. Paris : Ministère de l'Éducation nationale, coll. «Les Dossiers », n० 190.

BONNIOT-PAQUIEN N., COGÉRINO G. \& CHAMPELY S. (2009). "Les enseignants d'EPS face aux élèves qui décrochent de l'activité : interventions selon le sexe des élèves et discours relatifs aux comportements observés ». STAPS, n० 84, p. 77-92.

BORDES P. (2000). L'organisation de la classe d'éducation physique. Modalités de regroupement, relations interpersonnelles et progrès des élèves. Thèse de doctorat, sociologie, université Paris 5.

CLEUZIOU J.-P. (1996). “ Mesures de l'asymétrie et cotation des performances ». Sciences et motricité, no 28 , p. 44-52.
CLEUZIOU J.-P. (2000). “ L'analyse du menu et des notes ". In B. David (dir.), Éducation physique et sportive: la certification au baccalauréat. Paris : INRP, p. 77-124.

COGÉRINO G. (2006). «La mixité en éducation physique et sportive : de la gestion des groupes à une réflexion sur l'équité ». In G. Cogérino (dir.), La mixité en éducation physique. Paroles, réussites, différenciations. Paris : Éd. Revue EP.S, n॰ 67, p. 9-27.

COLTICE M. (2004). «En danse, débloquer les stéréotypes ». Contre Pied, no 15, p. 51-53.

COMBAZ G. \& HOIBAN O. (2008). « Le rôle de l'école dans la construction des inégalités de sexe ». Travail, genre et sociétés, n²0, p. 129-150.

COUCHOT-SCHIEX S. \& TROTTIN B. (2005). «Interactions enseignants/élèves : variations en fonction du sexe et du genre ». In G. Cogérino (dir.), Filles et garçons en EPS. Paris : Éd. Revue EP.S, p. 163-182.

CRAHAY M. (2000). L'école peut-elle être juste et efficace? Bruxelles : De Boeck Université.

DAVIS K. (2003). « Teaching for gender equity in physical education ". Women in sport and physical education, no $12(2)$, p. 55-82. 
DAVISSE A. \& VOLONDAT M. (1987). « Mixité, pédagogie des différences et didactique ". Revue EP.S, n० 206, p. 53-56.

DEPRAZ N. (2002). La conscience. Approches croisées, des classiques aux sciences cognitives. Paris : Armand Colin.

DUBET F. (2004). L'école des chances : qu'est ce qu'une école juste ? Paris : Éd. du Seuil.

DUBET F. \& DURU-BELLAT M. (2004). «Qu'est-ce qu'une école juste ? ". Revue française de pédagogie, n 146, p. 105-114.

DURU-BELLAT M. (1990). L'école des filles, quelle formation pour quels rôles sociaux ? Paris : L'Harmattan.

DURU-BELLAT M. (1994). “Filles et garçons à l'école, approches sociologiques et psychosociales. 1. Des scolarités sexuées, reflet de différences d'aptitude, ou de différences d'attitudes ? ". Revue française de pédagogie, no 109, p. 111-141.

DURU-BELLAT M. (1995). «Filles et garçons à l'école, approches sociologiques et psychosociales. 2. La construction scolaire des différences entre les sexes". Revue française de pédagogie, no 110, p. 75-109.

DURU-BELLAT M. (1999). " La sociologie des inégalités à l'école. Entre "engagement et distanciation" ". In D. Meuret, La justice du système éducatif. Bruxelles : De Boeck, p. 21-36.

EVANS J., DAVIES B. \& PENNEY D. (1996). « Teachers, teaching and the social construction of gender relations ". Sport, education and society, vol. 1, n० 2, p. 165-183.

FÉRNANDEZ-BALBOA J.-M. (1993). « Sociocultural characteristics of the hidden curriculum in physical education ". Quest, no 45, p. 230-254.

FONTAYNE P., SARRAZIN P. \& FAMOSE J.-P. (2001). " Les pratiques sportives des adolescents : une différenciation selon le genre ». STAPS, n० 55, p. 23-37.

GOFFMAN E. (1977). "The arrangement between the sexes ». Theory and society, vol. 4, no 3, p. 301-331.

GUÉRANDEL C. \& BEYRIA F. (2010). « La mixité dans les cours d'EPS d'un collège en ZEP : entre distance et rapprochement des sexes ". Revue française de pédagogie, no170, p. 17-30.

HADJI C. (2008). "De l'“éducativité” en éducation, et de son évaluation ". Communication au colloque Efficacité et équité en éducation organisé à Rennes du 19 au 21 novembre 2008.

HAUW D. (2000). "Les groupes en EPS ». In J.-P. Rey (dir.), Le groupe. Paris : Éd. Revue EP.S, p. 89-104.

KNOPPERS A. (1988). «Equity for excellence in physical education ". Journal of physical education, recreation and dance, no 59(6), p. 54-58.

KOIVULA N. (2001). « Perceived characteristics of sports categorised as gender neutral, feminine and masculine». Journal of sport behavior, n०24, p. 377-393.

LABERGE S. (2004). « Les rapports sociaux de sexe dans le domaine du sport: perspectives féministes marquantes des trois dernières décennies ». Recherches féministes, vol. 17 , no 1 , p. 9-38.
LEE V. E., MARKS H. M. \& BYRD T. (1994). « Sexism in single-sex and coeducational independent secondary school classrooms ". Sociology of education, vol. 67, n० 2, p. 92-120.

LIOTARD P. (2000). " Compréhension du corps et dénonciation du sport, 1968-1979 ». In T. Terret (dir.), Éducation physique, sport et loisir. 1970-2000. ClermontFerrand: Éd. AFRAPS, p. 121-138.

MOSCONI N. (1989). La mixité dans l'enseignement secondaire, un faux semblant. Paris : PUF.

MOUCHET A. (2003). Caractérisation de la subjectivité dans les décisions tactiques des joueurs d'élite1 en rugby. Thèse de doctorat, sciences et techniques des activités physiques et sportives, université Bordeaux 2.

RQNHOLT H. (2002). " "It's only the sissies...": analysis of teaching and learning process in physical education: a contribution to the hidden curriculum ". Sport, education and society, vol. 7, n० 1, p. 25-36.

TERRET T., COGÉRINO G. \& ROGOWSKI I. (2006). La mixité en EPS : pratiques et représentations. Paris : Éd. Revue EP.S.

THÉBERGE N. (2000). "Gender and sport 》. In J. J. Coakley \& E. Dunning (dir.), Handbook of sport studies. Londres: Sage publications, p. 323-335.

THEUREAU J. (2004). Le cours d'action : méthode élémentaire. Toulouse : Octarès.

VERMERSCH P. (1994). L'entretien d'explicitation en formation initiale et en formation continue. Paris : ESF.

VERMERSCH P. (1998). « Husserl et l'attention 1. L'attention comme mutations de l'intentionnalité ». Expliciter, n॰24, p. 7-23.

VERMERSCH P. (2002). " La prise en compte de la dynamique attentionnelle : éléments théoriques ". Expliciter, no 43, p. 27-39.

VERSCHEURE I. (2009). «Modalités de direction d'étude et apprentissage de l'attaque en volley-ball : quels effets de genre? ». eJRIEPS, no 18, p. 122-155.

VERSCHEURE I. \& AMADE-ESCOT C. (2007). "The gendered construction of physical education content as the result of the differentiated didactic contract ". Physical Education and Sport Pedagogy, no 12(3), p. 245272.

VIGNERON C. (2006a). "Sports collectifs : de l'ambition pour les filles ». In G. Cogérino (dir.), La mixité en éducation physique. Paroles, réussites, différenciations. Paris : Éd. Revue EP.S, n॰ 67, p. 55-90.

VIGNERON C. (2006b). "Les inégalités de réussite en EPS entre filles et garçons : déterminisme biologique ou fabrication scolaire ? ". Revue française de pédagogie, no 154, p. 111-124.

WRIGHT J. (1997). " The construction of gendered contexts in singles ex and coeducational physical education lessons ". Sport, education and society, vol. 2, no 1, p. 55-72. 
ANNEXE 1. PANEL DES ENSEIGNANT-E-S CHOISI-E-S

\begin{tabular}{|c|c|c|c|c|}
\hline Sexe & Âge & Ancienneté & Diplôme & Établissement \\
\hline \multirow{5}{*}{$\begin{array}{l}12 \text { femmes } \\
12 \text { hommes }\end{array}$} & $\begin{array}{l}6 \text { E de moins de } \\
30 \text { ans }\end{array}$ & $\begin{array}{l}4 \text { E de moins de } 5 \text { ans } \\
\text { d'ancienneté }\end{array}$ & $\begin{array}{l}2 \text { agrégé-e-s } \\
\text { externes }\end{array}$ & \multirow{5}{*}{$\begin{array}{l}14 \text { collèges non REP } \\
10 \text { collèges REP }\end{array}$} \\
\hline & $10 \mathrm{E}$ de 31 à 40 ans & $\begin{array}{l}4 \text { E de } 6 \text { à } 10 \text { ans } \\
\text { d'ancienneté }\end{array}$ & $\begin{array}{l}4 \text { agrégé-e-s } \\
\text { internes }\end{array}$ & \\
\hline & $4 \mathrm{E}$ de 41 à 50 ans & $\begin{array}{l}10 \text { E de } 11 \text { à } 20 \text { ans } \\
\text { d'ancienneté }\end{array}$ & $\begin{array}{l}14 \text { CAPEPS } \\
\text { externes }\end{array}$ & \\
\hline & $\begin{array}{l}4 \text { E de plus de } \\
51 \text { ans }\end{array}$ & $\begin{array}{l}2 \text { E de } 21 \text { à } 30 \text { ans } \\
\text { d'ancienneté }\end{array}$ & $\begin{array}{l}4 \text { CAPEPS } \\
\text { internes }\end{array}$ & \\
\hline & & $\begin{array}{l}4 \text { E de plus de } 30 \text { ans } \\
\text { d'ancienneté }\end{array}$ & & \\
\hline
\end{tabular}

ANNEXE 2. RÉPARTITION DES ACTIVITÉS CHOISIES PAR LES ENSEIGNANT-E-S SELON LE TYPE DE MIXITÉ

\begin{tabular}{|c|c|c|c|c|c|}
\hline & \multirow{2}{*}{$\begin{array}{l}\text { Nombre de moments } \\
\text { évoqués dans les EdE } \\
\text { (sans les } 4 \text { moments } \\
\text { indexés } 2 \text { fois) }\end{array}$} & \multicolumn{4}{|c|}{$\begin{array}{l}\text { Types de mixités évoquées en fonction des } \\
\text { activités physiques }\end{array}$} \\
\hline & & $\begin{array}{l}\text { Mixité } \\
\text { séparée }\end{array}$ & $\begin{array}{l}\text { Mixité } \\
\text { banalisée }\end{array}$ & $\begin{array}{l}\text { Mixité } \\
\text { recherchée }\end{array}$ & $\begin{array}{l}\text { Mixité } \\
\text { réfléchie }\end{array}$ \\
\hline $\begin{array}{l}\text { Activités masculines: } \\
\text { rugby, football, handball, } \\
\text { basket-ball, tchoukball, lutte, } \\
\text { boxe, judo, javelot }\end{array}$ & 19 & 6 & 4 & 7 & 2 \\
\hline $\begin{array}{l}\text { Activité attribuées aux deux } \\
\text { sexes : } \\
\text { badminton, tennis de table, } \\
\text { course d'orientation, volley-ball, } \\
\text { ultimate, roller }\end{array}$ & 12 & 1 & 6 & 4 & 3 \\
\hline $\begin{array}{l}\text { Activités féminines: } \\
\text { acrosport, danse }\end{array}$ & 8 & 0 & 3 & 5 & 2 \\
\hline
\end{tabular}

Note : ces chiffres tiennent compte des 4 moments indexés dans deux catégories de mixité. 